\title{
Fiber coupled ultrafast scanning tunneling microscope
}

\author{
Keil, Ulrich Dieter Felix; Jensen, Jacob Riis; Hvam, Jørn Märcher
}

Published in:

Journal of Applied Physics

Link to article, DOI:

10.1063/1.364322

Publication date:

1997

Document Version

Publisher's PDF, also known as Version of record

Link back to DTU Orbit

Citation (APA):

Keil, U. D. F., Jensen, J. R., \& Hvam, J. M. (1997). Fiber coupled ultrafast scanning tunneling microscope. Journal of Applied Physics, 81(7), 2929-2934. https://doi.org/10.1063/1.364322

\section{General rights}

Copyright and moral rights for the publications made accessible in the public portal are retained by the authors and/or other copyright owners and it is a condition of accessing publications that users recognise and abide by the legal requirements associated with these rights.

- Users may download and print one copy of any publication from the public portal for the purpose of private study or research.

- You may not further distribute the material or use it for any profit-making activity or commercial gain

- You may freely distribute the URL identifying the publication in the public portal

If you believe that this document breaches copyright please contact us providing details, and we will remove access to the work immediately and investigate your claim. 


\title{
Fiber coupled ultrafast scanning tunneling microscope
}

\author{
Ulrich D. Keil, ${ }^{\text {a) }}$ Jacob R. Jensen, and Jørn M. Hvam \\ Mikroelektronik Centret, DTU building 345 east, DK-2800 Lyngby, Denmark
}

(Received 22 October 1996; accepted for publication 16 December 1996)

\begin{abstract}
We report on a scanning tunneling microscope with a photoconductive gate in the tunneling current circuit. The tunneling tip is attached to a coplanar transmission line with an integrated photoconductive switch. The switch is illuminated through a fiber which is rigidly attached to the switch substrate. By using a firmly attached fiber we achieve an excellent reproducibility and unconstrained positioning of the tip. We observe a transient signal with 2.9 ps pulse width in tunneling mode and $5 \mathrm{ps}$ in contact mode. The instrument is applied to investigating the mode structure on a coplanar waveguide. The measurements show that the probe works as a transient voltage detector in contact and a capacitively coupled transient field detector in tunneling mode. We do not measure the transient voltage change in the ohmic tunneling current. In this sense, the spatial resolution for propagating electrical pulses is better in contact mode than in tunneling mode.

(C) 1997 American Institute of Physics. [S0021-8979(97)00407-6]
\end{abstract}

\section{INTRODUCTION}

The first realization of picosecond time resolution with a photoconductively gated scanning tunneling microscope was reported by Weiss et al. in 1993. ${ }^{1,2}$ Meanwhile their results have been confirmed by two other groups $s^{3,4}$ and subpicosecond time resolution has been achieved. ${ }^{5}$ The simultaneous spatial resolution is still subject to controversy. ${ }^{6}$ The limits of simultaneous spatial and temporal resolution have not been measured directly, yet. A linear dependence of the signal amplitude on the de tunneling current, or gap conductance, has been observed so far consistently. Assuming an exponential relation between tunneling current and distance, this dependence can also be seen as an exponential decrease with distance. The observed signal shapes range from a pure derivative of the measurement in contact to a resemblance of the contact measurement with a shortened response. Both results indicate a capacitive coupling. The exponential distance dependence is intriguing because it suggests that the spatial resolution for high frequency signals scales in the same way as for dc signal. However, models based on this assumption could only explain the measurements with a disappearing geometrical capacitance of the tunneling gap. ${ }^{2}$ Recently, the exponential amplitude dependence has been explained as an effect of the measurement technique while taking into account a geometric capacitive. ${ }^{6}$ For interpreting the results it has to be remembered that in a sampling technique the signals are not measured in real time, but the time resolution is achieved by changing the delay between pump and probe times. The observed transient signal in the current is a time averaged, delay dependent current. A dc (or low frequency) current can, however, only be drawn through the ohmic part of the impedance and must, in our specific case (in lieu of another current path), be controlled by the gap resistance. Thus, although the signal shape can be determined by the gap capacitance the amplitude is set by the gap

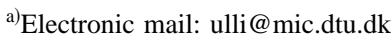

resistance. We will discuss how these issues can be resolved in our version of an ultrafast scanning tunneling microscope (USTM).

The instrument is based on the concept of a USTM presented in Ref. 1. Our approach differs in two aspects from the previously reported designs. ${ }^{1,3}$ First, we attach a fiber directly to the photoconductive switch. This illumination principle enables an unconstrained positioning and increased scanning range of the tip by avoiding a scanning position dependent illumination of the photoconductive switch. Secondly, the tunneling tip is attached to a coplanar strip line (CPS) which provides direct electrical access to the tip for monitoring the dc tunneling current. In addition, the CPS ensures a low attenuation of the high frequency component of the signal. As a test of the instrument, we measure the mode structure of an electrical pulse generated on a coplanar waveguide (CPW). By illuminating an in-line photoconductive switch, signal and ground lines are distinguished and the main voltage pulse is confined to the center line. In this setup, we can measure the coupling from the signal line while the tip is in tunneling mode or in direct contact with the ground lines. In this paper we refer to the measured signal as a "transient signal in tunneling mode." With this terminology we do not imply whether the signal is of resistive or capacitive origin.

\section{EXPERIMENT}

\section{A. General setup}

Figure 1 shows a sketch of the setup. The base instrument used in these investigations is a commercial ambient STM (DME Rasterscope). The laser source is a mode-locked Ti:sapphire laser with a pulse width of $100 \mathrm{fs}$ and a wavelength of $800 \mathrm{~nm}$. The laser beam is split into pump and probe beams. The pump beam generates the electrical pulse on the sample and the probe beam gates the current on the probe. The optical delay of the probe beam determines the gating time and provides the time resolution. In all measurements both pump beam and probe beam are chopped and the modulation of the probe current is measured with a lock-in 


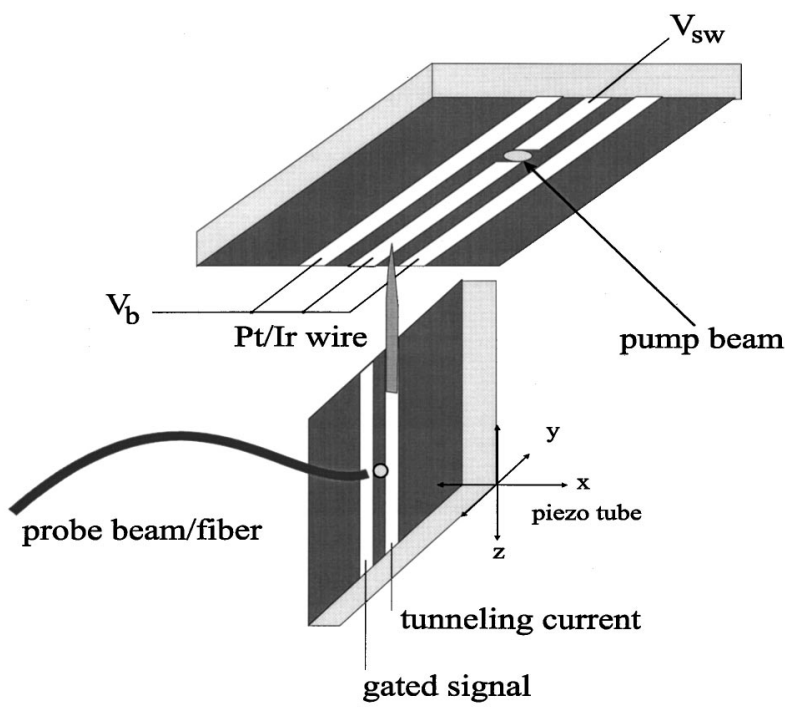

FIG. 1. Schematic setup of the USTM.

amplifier locked to the difference frequency. The laser beams are modulated by acousto-optic modulators and the maximum modulation frequency is $5 \mathrm{MHz}$. The test sample is mounted above the tip and can be manually aligned. A subpicosecond electrical pulse is generated by illuminating an in-line photoconductive switch on a CPW. The pulse is picked up by the gated tunneling tip which is mounted on the piezo scanner tube.

\section{B. Probe tip design and optimization}

The gated tip consists of a Pt/Ir wire with a $5 \mu \mathrm{m}$ diameter which is glued to a CPS with conducting silver epoxy. Sample and probe transmission lines are 300-400-nm-thick $\mathrm{Ti} / \mathrm{Au}$ contacts deposited on low-temperature grown GaAs (LT GaAs) substrates. Width and spacing of the probe CPS contacts are $10 \mu \mathrm{m}$. The small dimensions enable the transmission of frequencies up to the THz regime. ${ }^{7}$ The plane of the CPS on the probe is kept parallel to the propagation direction on the sample transmission line. Hence, the pick-up of far-field $\mathrm{THz}$ radiation from the sample by the probe transmission line is minimized. The photoconductive switch on the probe is excited through a fiber. The fiber is aligned over the gap of the transmission line for maximum photocurrent. In this position the fiber is permanently glued in place. The photoconductive switch is formed wherever the probe light illuminates the gap between the lines. By using this sliding-contact switch ${ }^{8}$ we avoid an impedance discontinuity as it would be formed by an in-line photoconductive switch. The first source of reflection is the unterminated end of the transmission line about $2 \mathrm{~mm}$ away. This distance is equivalent to a propagation time of $40 \mathrm{ps}^{7}$

The growth and annealing procedure for both tip and sample are optimized for a fast response. The use of LT GaAs instead of ion-irradiated SOS results in an increased sensitivity. ${ }^{9}$ The $1-\mu$ m-thick LT GaAs layer is grown at $250{ }^{\circ} \mathrm{C}$ and subsequently annealed for one minute by rapid thermal annealing at $T_{a}=525{ }^{\circ} \mathrm{C}$ or $600{ }^{\circ} \mathrm{C}$. For first characterization, differential transmission measurements are per-

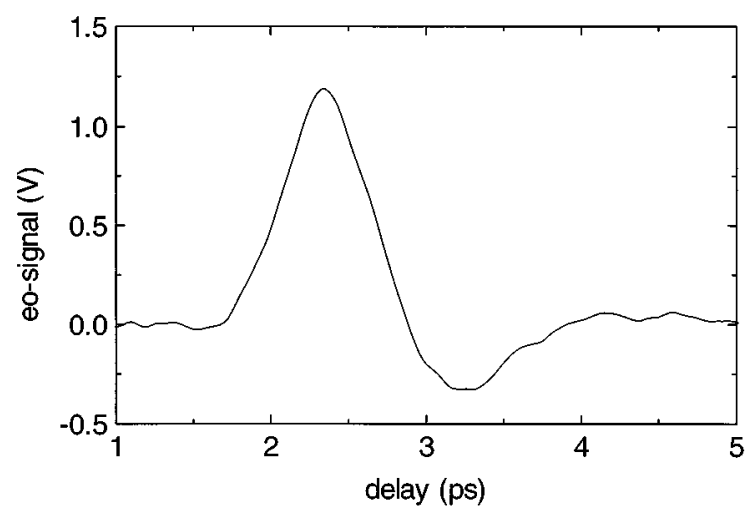

FIG. 2. Electrical pulse measured on the sample CPW by electro-optic sampling $V_{\mathrm{sw}}=10 \mathrm{~V}$. The LT GaAs substrate is grown at $250{ }^{\circ} \mathrm{C}$ and annealed at $600{ }^{\circ} \mathrm{C}$.

formed on a LT GaAs layer separated from the substrate by epitaxial lift-off. ${ }^{10}$ These measurements show that the carrier life time increases to about 800 fs for $T_{a}=600{ }^{\circ} \mathrm{C}$ and is below our temporal resolution limit of about $200 \mathrm{fs}$ for $T_{a}=525^{\circ} \mathrm{C}$. Other important measures of the substrate are the conductivity and the breakdown field. For a CPS with width and spacing of $5 \mu \mathrm{m}$ and a length of $3 \mathrm{~mm}$, the dark resistance from one line to the other is about $5 \mathrm{M} \Omega$ for $T_{a}=560{ }^{\circ} \mathrm{C}$ and $60 \mathrm{M} \Omega$ for $T_{a}=600{ }^{\circ} \mathrm{C}$. The latter value is too high for a photoconductive switch which is in series with the tunneling current resistance. The annealing temperature for the probe substrate is therefore chosen to be $560{ }^{\circ} \mathrm{C}$. For the sample, however, the higher annealing temperature resulting in a higher breakdown field enables us to apply a higher voltage to the pulse-generating switch. The higher switch voltage, in turn, results in a higher amplitude of the voltage pulse. Thus, the sample substrate is annealed at $600{ }^{\circ} \mathrm{C}$. Electro-optic sampling ${ }^{11}$ measurements show a full width half maximum (FWHM) pulse width of 800 fs (Fig. 2 ). For a switch voltage, $V_{\mathrm{sw}}=10 \mathrm{~V}$, the pulse amplitude is 1 $\mathrm{V}$. Although this pulse is measured in a separate setup, it is typical for the input signal in the USTM, in amplitude as well as in pulse width.

As mentioned, we use an optical fiber attached directly to the photoconductive switch for gating the tunneling current. This way we achieve an increased scanning range and an excellent reproducibility of the tip behavior. A singlemode fiber is used to minimize dispersion of the optical pulse and the related decrease in time resolution. Using a firmly attached fiber on the one hand ensures an unrestricted positioning of the tip. On the other hand, the fiber also represents an additional noise source. We test, therefore, the spatial resolution achieved with this modified instrument. Figure 3 shows a high resolution image of a gold surface of the transmission line sample in constant current mode indicating a spatial resolution of $10-20 \mathrm{~nm}$. We find that the main limitation for the spatial resolution is given by the length of the tip wire (apart from the limitations set by scanning in air). Typical wire lengths are $100 \mu \mathrm{m}$. This length does not present a principle limit in our setup but wires of this length seem to tolerate crashing into the substrate better than shorter wires. 


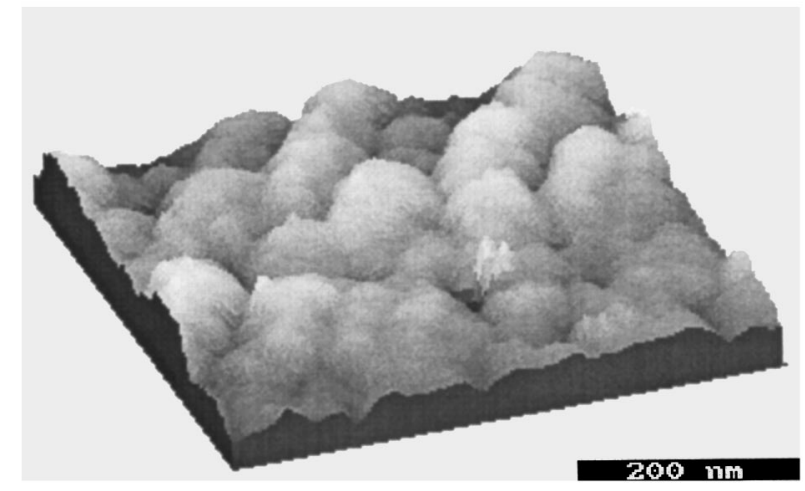

FIG. 3. Testing the spatial resolution of the modified tip, STM image of the surface of a gold transmission line in constant current mode. The black bar represents a length of $200 \mathrm{~nm}$.

\section{RESULTS AND DISCUSSION}

The test structure is a CPW with $5 \mu \mathrm{m}$ contact spacing and width (see Fig. 1). All three lines at the position of the tunneling tip are kept at a bias voltage, $V_{b}$, of around $50 \mathrm{mV}$ to supply a dc tunneling current. The switch voltage, $V_{\mathrm{sw}}$, (typically $2-10 \mathrm{~V}$ ) is applied to the other side of the in-line switch. Figure 4 shows the time resolved signals measured with the USTM. First the signal is measured while the tip is in contact with the center CPW electrode $\left(I_{\text {contact }}\right)$. Then the tip is retracted while a stable average tunneling current, $I_{\mathrm{dc}}$ (here $I_{\mathrm{dc}}=1.5 \mathrm{nA}$ ), is established. For this measurement the current modulation and the average current were measured with the internal pre-amp of the STM. For comparison a derivative of $I_{\text {contact }}$ is included in the graph. All curves are normalized to one.

The transient signal in tunneling mode, $I_{\text {tunnel }}$, has an amplitude that is about a factor of 100 smaller compared to the contact measurement. Like the tunneling mode signals observed in Ref. 1, we observe a derivative of the transient voltage signal. The rise time $(10 \%-90 \%)$ of the tunneling mode signal is $2.6 \mathrm{ps}$ and the FWHM is $2.9 \mathrm{ps}$. The rise time of the contact signal is 2.7 ps and FWHM 5.2 ps. As in Refs.

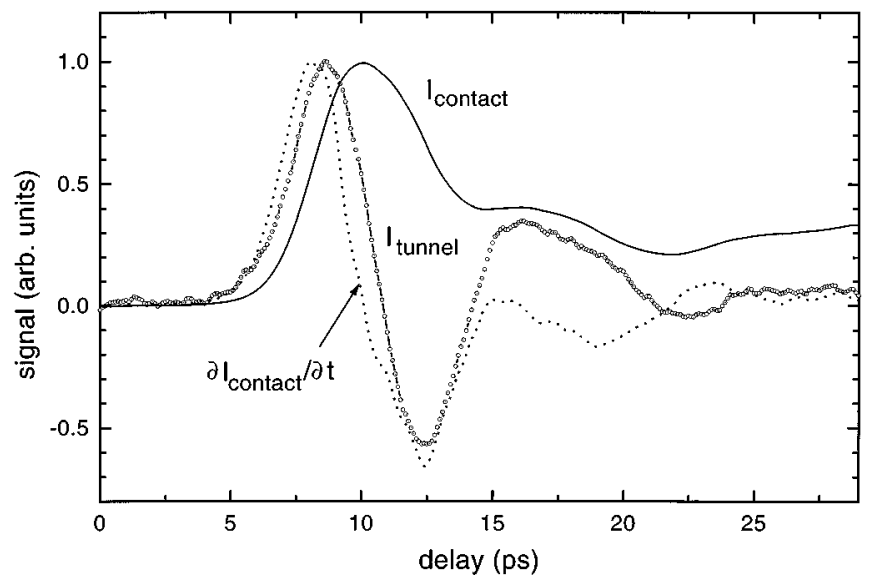

FIG. 4. Comparison of the measurement in contact, $I_{\text {contact }}$, the derivative, $\partial I_{\text {contact }} / \partial t$, and the measurement in tunneling mode, $I_{\text {tunnel }}, I_{\text {tunnel }}$ is measured with an average tunneling current of $1.5 \mathrm{nA}$. All signals are normalized.
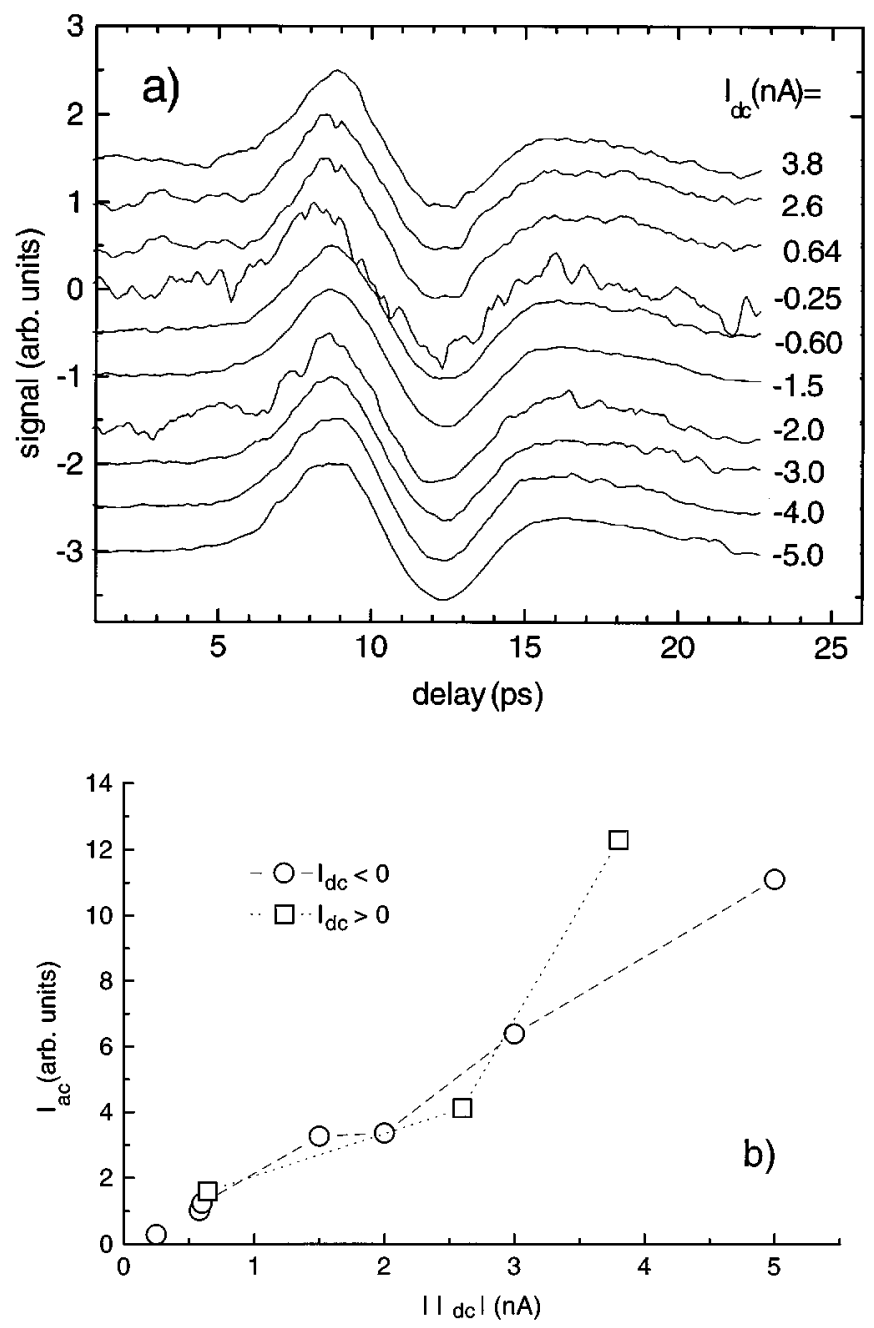

FIG. 5. Comparison of tunneling mode measurements for different average tunneling currents, $I_{\mathrm{dc}}$. All signals are normalized and an offset is added. The dependence of the maximum amplitude (at $t \approx 9 \mathrm{ps)}$ on $I_{\mathrm{dc}}$ is shown in (b).

1 and 3, we observe a linear relation between the dc tunneling current (over a range of $0.25-5 \mathrm{nA}$ ) and the amplitude of the transient signal (Fig. 5). We measure a signal shape that is completely independent of the magnitude and the polarity (with respect to the transient signal) of the dc tunneling current. Due to the good signal-to-noise ratio in these measurements we can also carefully investigate differences between the derivative of the contact signal, $\partial I_{\text {contact }} / \partial t$, and $I_{\text {tunnel }}$. These two signals start to differ noticeably from each other at a time delay of 5 ps after the initial peak (15 ps delay in Fig. 4).

Before interpreting the results by means of an equivalent circuit we point out some experimental details specific for this measurement. The average current and the modulated current were measured with a single pre-amp. The amplifier was connected to the tip through the photoconductive switch (left strip in Fig. 1) and the line with the tip was floating. (Floating here means that the tip is only connected through the substrate to virtual ground of the pre-amp.) The amplifier is the standard current voltage converter of the commercial STM instrument. Pump and probe beams were modulated at 

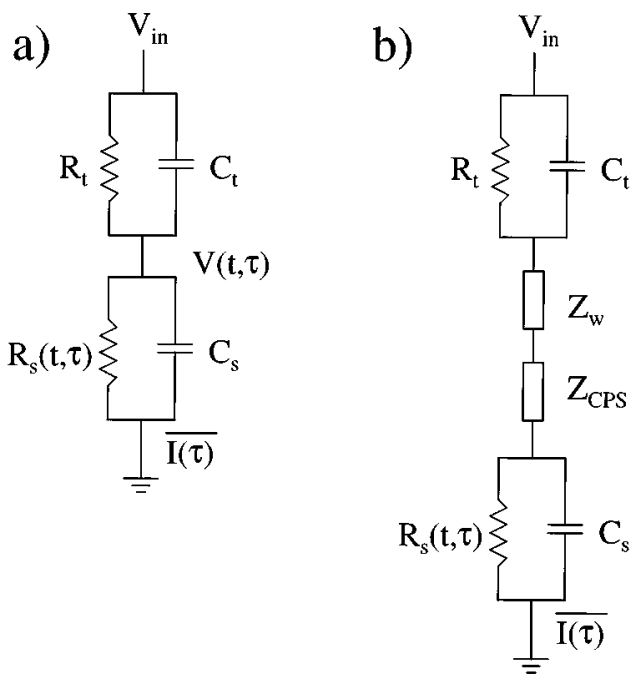

FIG. 6. Equivalent circuits: (a) photoconductive switch and tunneling junction as lumped elements (b) photoconductive switch and tunneling junction separated by tip wire $\left(Z_{w}\right)$ and transmission line impedance $\left(Z_{\mathrm{CPS}}\right) . V_{\text {in }}$ is the input signal on the sample CPW, $R_{t}$, the tunneling resistance, $C_{t}$, the capacitance of the tunneling junction, $R_{s}(t, \tau)$ the real and delay time dependent switch resistance, and $C_{s}$, the switch capacitance.

about $4 \mathrm{kHz}$. The modulation frequencies were kept above the cut-off frequency of the feedback loop and below the cut-off frequency of the pre-amp.

For the interpretation of the signals we consider the equivalent circuit depicted in Fig. 6. For closely spaced tunneling junction and photoconductive switch, junction and switch can be regarded as lumped elements [Fig. 6(a)]. In our case the time resolution of about 2 ps is smaller than the propagation time of approximately 3 ps (for a total distance of $300 \mu \mathrm{m}$ ) between junction and photoconductive switch. In this case [Fig. 6(b)] the characteristic impedances of the tip wire, $Z_{w}$, and the CPS, $Z_{\mathrm{CPS}}$, have to be considered. ${ }^{12}$ If the capacitive contribution at the tunneling junction is dominating, then $C_{t}$ and $Z_{w}$ form a differentiator. In contact mode the ohmic contribution is dominating and $R_{t}$ and $Z_{w}$ form a voltage divider or attenuator. The transitions from tunneling or contact junction to wire and wire to CPS both form impedance mismatches resulting in attenuation and reflection of the signal. The delayed pulse reflected at the $Z_{w} / Z_{\mathrm{CPS}}$ transition and then at the $Z_{w} / R_{t} C_{t}$ transition is in fact observed in Fig. 4 as a second bump in the contact measurement. In this sense, also the deviation from the derivative for longer delay times can be interpreted. The reflection at the $Z_{w} / R_{t} C_{t}$ should be stronger in the tunneling case where $R_{t}$ is significantly bigger than $Z_{w}$. The reflected signal is, therefore, more pronounced in the tunneling measurement.

The measurement shown in Fig. 7 is taken with a modified setup using two preamplifiers. Here, the gated current is measured with a second, external, pre-amp. The internal preamp controlling the feedback loop is directly connected to the tunneling tip via the other strip of the transmission line (see Fig. 1). In this case the tip is not floating, but connected to virtual ground of the internal pre-amp. Figure 7 shows that the transients for contact mode, contact mode derivative, and tunneling mode behave qualitatively exactly like the mea-

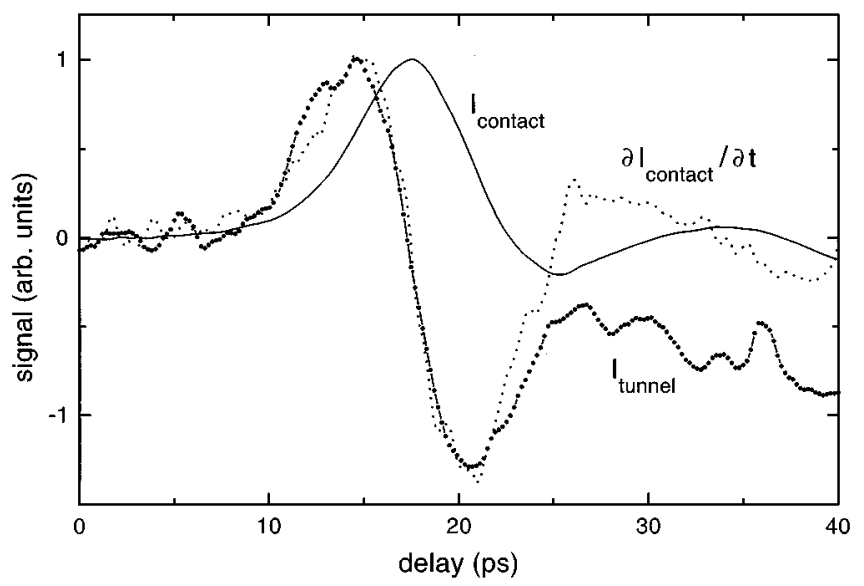

FIG. 7. Measurement with two preamplifiers. The internal preamplifier, controlling the feedback loop, is connected to the tip-side of the transmission line (right side in Fig. 1). A second preamplifier, measuring the modulated signal, is connected to the other side of the PCS. $I_{\text {contact }}, \partial I_{\text {contact }} / \partial t$, and $I_{\text {tunnel }}$ are shown for comparison. All signals are normalized.

surements with a floating tip in Fig. 4. However, first results show that the amplitude dependence observed in Fig. 5(b) disappears. Groeneveld et al. ${ }^{6}$ have pointed out that the floating tip explains the observed amplitude dependence. The unmodified detection scheme [as depicted in Fig. 6(a) and 6(b)] enables only the measurement of a dc current and this dc current can only be drawn through $R_{t}$. By connecting a second pre-amp, additional current paths become possible. These experiments are in progress and will be reported separately. ${ }^{13}$

In the following we use again the "floating tip" setup. In contact mode the gated tip represents a freely positionable photoconductive tip. ${ }^{14}$ Particularly in this mode, the fiber illumination of the photoconductive switch enables the measurements of voltage transients spatially resolved. We demonstrate this ability by measuring the mode structure of an electrical pulses which is, again, generated by optically exciting the in-line gap in a CPW. In the measurements shown in Fig. 8 the CPW has a contact width and spacing of $5 \mu \mathrm{m}$ and the tip is moved by $10 \mu \mathrm{m}$ perpendicular to the lines between the measurements. Figure 8(a) shows the measurement in contact mode and Fig. 8(b) in tunneling mode. As expected, the signal on the center (or signal) line is the strongest. The asymmetry between top and bottom electrodes shows that part of the signal is generated by photo induced carriers between the lines and not just in the in-line gap. This measurement indicates that the pump beam was not exactly centered on the CPW. For the measurement in tunneling mode only bottom and center line measurements are shown. Again the signal is strongest on the center line. Qualitatively, both measurements resemble the derivative of the respective contact measurement. However, the amplitude ratio (center to bottom signal) changed from a factor of 5 in contact to a factor of 2 in tunneling. This result indicates that in tunneling mode part of the signal is picked up from the center line. Although this interpretation requires further investigation, it means that the spatial resolution is, in this setup, better in the contact measurement and that in the tunneling measurement 

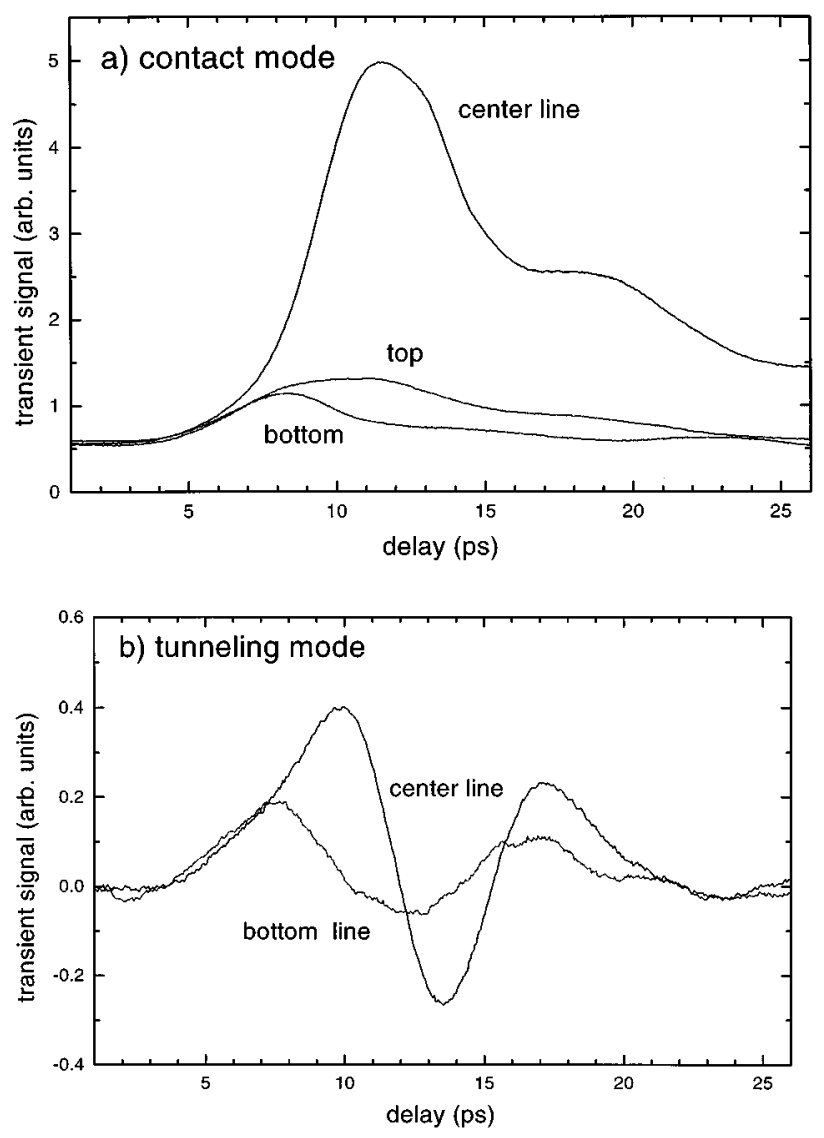

FIG. 8. Position dependent measurements on CPW: (a) in contact mode, (b) in tunneling mode. The amplitude ratios for center to bottom electrode signals are 5 in contact mode and 2 in tunneling mode.

part of the signal is capacitively coupled from the center line to the tunneling tip hovering above the bottom line. An interesting result is that the amplitude observed in tunneling mode is a factor of 2 larger on the center line compared to the bottom line. This is also the ratio in which the field is distributed on a CPW for dc bias or a TEM mode. This behavior indicates that the probe, in fact, acts as a "farfield" electric field probe and does not measure a voltage change localized in the tunneling region. In other words, the lateral (perpendicular to propagation) spatial resolution for voltage pulses on transmission lines is better in contact mode than in tunneling mode.

Another measurement that resolves the capacitive nature of the signal is shown in Fig. 9. Here the measurement was performed with a tip wire that was incidentally bent. The wire was oriented parallel to the sample CPW with the tip pointing away from the gap, i.e., the contact or tunneling junction was further down the propagation path than the tip bend. If we now compare the derivative of the contact measurement with the tunneling measurement, we see a significant shift of the tunneling signal towards shorter delay times. The intuitive explanation for this result is that the signal in tunneling mode is capacitively picked up by the bent wire before the pulse reaches the tunneling region. This result demonstrates that the transient signal in tunneling mode is due to a capacitive coupling which is not necessarily local-

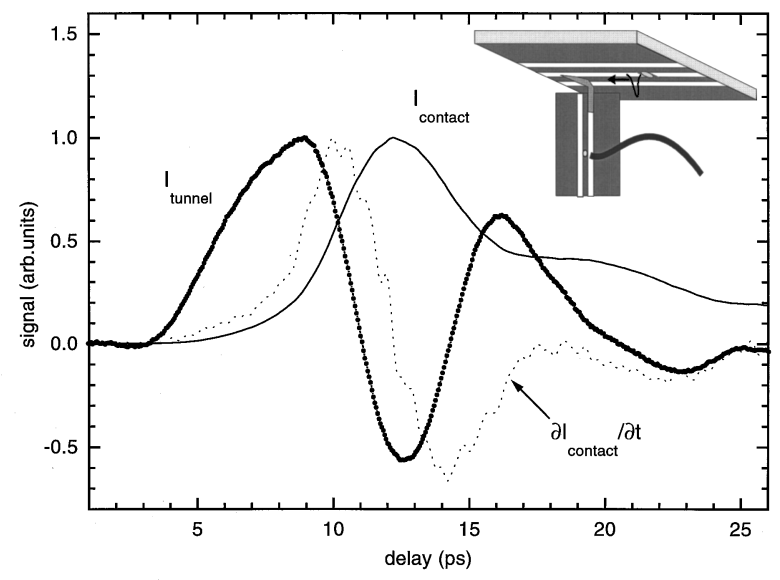

FIG. 9. Measurement with a tip bent in propagation direction (see inset). $I_{\text {tunnel }}$ exhibits a shift towards earlier delay times with respect to $I_{\text {contact }}$ and $\partial I_{\text {contact }} / \partial t$. All signals are normalized.

ized in the tunneling region. With the bent tip the tunneling regime and the main capacitive coupling regime can be spatially separated.

\section{SUMMARY}

We present a fiber coupled, photoconductively gated STM. We show that the spatial resolution with a fiber attached to the probe is on the order of 10-20 nm without simultaneous time resolution. We demonstrate and use the ability to freely position the tip on the sample. By having a fixed illumination of the probe photoconductive switch we achieve an excellent reproducibility of the tip behavior independently of the scanning position. The mode structure of a pulse propagating on a $\mathrm{CPW}$ can be resolved in contact mode.

The signal shape in tunneling mode resembles the derivative of the contact mode signal. This signal shape can be explained for a probe design where tunneling junction and photoconductive switch can not be considered as lumped elements, i.e., where the junction-switch propagation time exceeds the time resolution. By comparing the derivative of the contact signal with the tunneling signal we can identify a signal caused by reflection from the tunneling tip wire. For a "floating tip", we observe a linear relation between the dc tunneling current and the amplitude of the transient signal. First results show that this amplitude dependence disappears when the tip is directly connected to virtual ground of the current pre-amp. The qualitative signal shapes, however, are the same in both configurations. By comparing contact and tunneling measurement on adjacent electrodes in a coplanar waveguide, we observe the first direct evidence of the lateral spatial resolution limits of the USTM for measuring propagating electrical pulses. We do not observe evidence for a transient tunneling signal. All measurements can be explained by a long-range capacitive coupling. The coupling range in our case appears to be determined by the extension of the field in the sample. It would thus be interesting to test the instrument on a structure with a strongly confined electric field. 


\section{ACKNOWLEDGMENTS}

We would like to thank Claus Sørensen at the III/V Nanolab for the growth of the LT GaAs samples and Ole Hansen for fruitful discussions. This work is supported by the Danish Research Academy and by the Danish Ministries of Research and Industry in the framework of CNAST.

${ }^{1}$ S. Weiss, D. F. Ogletree, D. Botkin, M. Salmeron, and D. S. Chemla, Appl. Phys. Lett. 63, 2567 (1993).

${ }^{2}$ S. Weiss, D. Botkin, D. F. Ogletree, M. Salmeron, and D. S. Chemla, Phys. Status Solidi B 188, 343 (1995).

${ }^{3}$ R. H. M. Groeneveld, Th. Rasing, L. M. F. Kaufmann, E. Smalbrugge, J. H. Wolter, M. R. Melloch, and H. van Kempen, J. Vac. Sci. Technol. B 14, 861 (1996).

${ }^{4}$ U. D. Keil and J. M. Hvam, Tech. digest, EQEC' 96 , Hamburg, Germany, IEEE cat. no. 96TH8162, p. 202.
${ }^{5}$ D. Botkin, J. Glass, D. S. Chemla, D. F. Ogletree, M. Salmeron, and S. Weiss, Appl. Phys. Lett. 69, 1321 (1996).

${ }^{6}$ R. H. M. Groeneveld and H. van Kempen, Appl. Phys. Lett. 69, 2294 (1996).

${ }^{7}$ U. D. Keil, D. R. Dykaar, A. F. J. Levi, R. F. Kopf, L. N. Pfeiffer, S. B. Darack, and K. W. West, IEEE J. Quantum Electron. QE-28, 2333 (1992).

${ }^{8}$ M. B. Ketchen, D. Grischkowski, T. C. Chen, C.-C. Chi, I. N. Duling III, N. J. Halas, and J. M. Halbout, Appl. Phys. Lett. 48, 751 (1986).

${ }^{9}$ S. Gupta, J. F. Whitaker, and G. A. Mourou, IEEE J. Quantum Electron. QE-28, 2464 (1992).

${ }^{10}$ U. D. Keil, J. M. Hvam, S. Tautz, S. U. Dankowski, P. Kiesel, and G. H. Döhler, Appl. Phys. Lett. 70, 72 (1997).

${ }^{11}$ J. A. Valdmanis and G. Mourou, IEEE J. Quantum Electron. QE-22, 69 (1986).

${ }^{12}$ D. A. Botkin, Ph.D. thesis, UC Berkeley, 1995.

${ }^{13}$ U. D. Keil, J. R. Jensen, and J. M. Hvam (unpublished).

${ }^{14}$ J. Kim, S. Williamson, J. Nees, S. Wakana, and J. Whitaker, Appl. Phys. Lett. 62, 2268 (1993). 